Original Research

\title{
Characterization of Early Indicators of Cardiac Allograft Vasculopathy Lesions in a Rat Model Using Non-Invasive Cellular MR
}

Qing Ye ${ }^{1,2,{ }^{\dagger}}$, Yijen L. Wu ${ }^{1,3,4,{ }^{\dagger}}$, Lesley M. Foley ${ }^{1,5}$, Parker H. Mills ${ }^{1}$, Fang-Cheng Yeh ${ }^{1,6}$, Haosen Zhang ${ }^{1}$, Danielle F. Eytan ${ }^{1,7}$, Li Liu ${ }^{1,8}$, T. Kevin Hitchens ${ }^{1,5}$, Chien Ho ${ }^{1, *}$

1. Department of Biological Sciences, Carnegie Mellon University, Pittsburgh, PA, USA; E-Mails: parker.mills@gmail.com; zhanghaosen@hotmail.com; chienho@andrew.cmu.edu

2. Department of Neurology, University of Pittsburgh, Pittsburgh, PA, USA; E-Mail: qiy18@pitt.edu

3. Rangos Research Center Animal Imaging Core, Children's Hospital of Pittsburgh of University of Pittsburgh Medical Center, Pittsburgh, PA, USA; E-Mail: vijenwu@pitt.edu

4. Department of Developmental Biology, School of Medicine, University of Pittsburgh, Pittsburgh, PA, USA

5. Animal Imaging Center, University of Pittsburgh School of Medicine, Pittsburgh, PA, USA; E-Mail: Imf107@pitt.edu; tkh17@pitt.edu

6. Department of Neurological Surgery, University of Pittsburgh, Pittsburgh, PA, USA; E-Mail: frank.yeh@gmail.com

7. Otolaryngology-Head and Neck Surgery, Johns Hopkins Hospital, Baltimore, MD, USA; E-Mail: danielle.eytan@gmail.com

8. National Institute of Neurological Disorders and Stroke, National Institute of Health, Bethesda, MD, USA; E-Mail: li.liu3@nih.gov

$\dagger$ These authors contributed equally to this work.

* Correspondence: Chien Ho; E-Mail: chienho@andrew.cmu.edu

Academic Editor: Yasuhiko Sugawara

Special Issue: Perspectives on Heart Transplantation

OBM Transplantation

2019, volume 3, issue 3

doi:10.21926/obm.transplant.1903083
Received: January 07, 2019

Accepted: August 26, 2019

Published: August 29, 2019

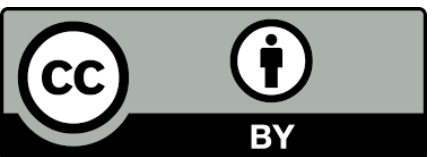

(C) 2019 by the author. This is an open access article distributed under the conditions of the Creative Commons by Attribution License, which permits unrestricted use, distribution, and reproduction in any medium or format, provided the original work is correctly cited. 


\begin{abstract}
Background: Cardiac allograft vasculopathy (CAV) remains a major obstacle to long-term heart allograft survival. A number of studies show that immune mechanisms are involved in CAV. Using non-invasive cellular MRI (CMRI) to explore indicators of CAV lesions and characterize its development could provide new insights into the target immune cells that are responsible for the progression of CAV and reveal early markers of the disease before irreversible changes occur.
\end{abstract}

Methods and Results: Rat heart transplant allografts $(n=88)$ and isografts $(n=22)$ were employed in this study. CMRI at 4.7-Tesla revealed a few hypointense foci in the graft early on during CAV with more regions becoming involved as the disease progressed. The areas of image hypointensity corresponded with infiltrating ED $1^{+}$cells labeled with micrometer-sized superparamagnetic iron oxide (MPIO), confirmed by MR microscopy (MRM) at 11.7-Tesla and pathology. MRI abnormalities of the transplanted hearts correlated well with histopathological findings. MPIO-labeled cells counted by a computer algorithm that analyzed 3D MRM volumes exhibited a strong correlation with immunohistochemical inspection of ED1 ${ }^{+}$cell infiltration counted manually $\left(R^{2}=0.8075\right)$. Histology found that in the early phases of CAV, lesions were focal and mostly began in the adventitia.

Immunohistochemistry indicated that the infiltrates were mostly $E D 1^{+}$cells and that their density was significantly correlated with the severity of CAV $(p<0.05)$.

Conclusions: This study illustrates the feasibility of monitoring MPIO-labeled ED ${ }^{+}$cells as an early indicator for CAV before vessel wall changes using non-invasive CMRI, which provides information on the entire heart.

\title{
Keywords
}

Rat heart transplantation; cardiac allograft vasculopathy; magnetic resonance imaging; iron oxide particles; immune cells

\section{Introduction}

Heart transplantation has become the preferred treatment for many patients with end-stage heart failure [1]. Although advances in immunosuppression have significantly reduced the incidence of acute rejection, chronic rejection, also known as cardiac allograft vasculopathy (CAV), remains a major problem and accounts for about $50 \%$ of mortality by year 10 post-transplant $[2,3]$. Early diagnosis of CAV is important for prevention and treatment of the disease. However, establishing such a diagnosis remains challenging [4], because the pathogenesis of CAV is not fully elucidated and the majority of CAV presents insidiously due to the lack of clinical symptoms of ischemia in the denervated allograft $[4,5]$. Current clinical practices for detecting CAV depend mostly on invasive techniques, such as endomyocardial biopsy, coronary angiography, and intravascular ultrasound (IVUS) [6], to assess coronary vascular changes. Once vessel narrowing or arterial wall thickening has developed sufficiently to be detected by coronary angiography or IVUS, treatments may be too late to rescue the graft, thus long-term survival is reduced significantly [4]. 
To date, non-invasive in-vivo observation of CAV lesions, prior to arterial wall thickening and luminal narrowing, has not been established.

The development of CAV is a complex process that is not fully understood, but a growing body of evidence supports the involvement of an inflammatory milieu that contributes to fibromuscular and smooth muscle cell proliferation with subsequent coronary obstruction [7-9]. It has also long been noted that mononuclear cells, mainly monocytes/macrophages, and T-cells, are abundant in coronary artery lesions and can orchestrate the development of CAV $[9,10]$. Infiltrating monocytes/macrophages are increasingly recognized as inflammatory amplifiers and are particularly appealing as pivotal and final effectors for CAV $[8,9]$, though the disease may result from a diverse array of actions in both the innate and adaptive immune responses [11, 12]. Thus, these infiltrates may be ideal targets to visualize CAV development.

Cellular MRI (CMRI) is a technique being used in both research and clinical settings for serially monitoring a myriad of events, such as inflammatory responses $[13,14]$ or cellular therapies in intact organisms and patients $[15,16]$. Cells of interest can be labeled either ex vivo or in vivo with an appropriate contrast agent, and their migration and accumulation can then be tracked by MRI [17-20]. In this study we used a rat transplantation pair with restricted antigenic specificity that develops chronic rejection without any immunologic manipulation [21]. We further used a working heterotopic cardiac transplantation $(\mathrm{WKHt})$ model where the recipient receives an additional heart and lung in the abdomen. This model preserves intact pulmonary circulation and physiological pressure and volume loading [22]. Our earlier work showed that recipient macrophages in a rat CAV model can be effectively labeled in vivo by phagocytosis of nano- and micrometer-sized paramagnetic iron oxide (USPIO and MPIO) particles [18]. The ability to successfully monitor target cells over a long period of time by CMRI has led us to attempt to map the accumulation pattern of MPIO-labeled cells as a marker for characterizing the development of CAV in order to determine the onset of lesions and identify the early stages of the disease.

\section{Materials and Methods}

\subsection{Animal Model and Experimental Designs}

Syngeneic transplantation (isograft, $\mathrm{n}=22$ ) was performed using PVG R.8 to PVG R.8. We examined 4 times more allograft hearts than isograft controls, because there were variations among the allografts in developing different degrees of CAV during the same time period after transplantation [21, 23]. At least 5 transplants per experimental group/per end-time point were performed. All rats weighed between 260 and $280 \mathrm{~g}$ at the time of transplantation and received humane care in compliance with the Guide for the Care and Use of Laboratory Animals published by the National Institutes of Health.

The WkHt model of heterotopic heart and lung transplantation was chosen for this study because orthotopic heart transplantation is not feasible at the present time and conventional abdominal heterotopic transplant hearts do not receive proper pressure preload, and the myocardium experiences atrophy over time [24]. The technical details of the surgical procedure of the WkHt model are described elsewhere $[18,25]$. Briefly, after the chest wall is opened in the donor, $500 \mathrm{U} / \mathrm{kg}$ body weight of sodium heparin is injected into the IVC, and the left lung is ligated and excised. The azygos vein with the left and right superior vena cava (SVC) is ligated and divided. 
The descending thoracic aorta is transected, and $10 \mathrm{ml}$ of cold lactated Ringer's solution (Abbott Laboratories) is infused into the IVC followed by ligation and division of the IVC. The ascending aorta is dissected and transected at the portion between the left common carotid artery and the left subclavian artery. The graft aorta and superior vena cava were anastomosed to the recipient aorta and IVC, respectively, in an end-to-side fashion with a continuous 8-0 polypropylene suture (Ethicon Inc, Somerville, NJ). The abdominal wall was then sutured with 6-0 silk (Ethicon Inc.). Graft survival was monitored daily after transplantation for 1 week and then weekly by palpating the transplanted heart.

Since the graft superior vena cava is anastomosed to the recipient abdominal inferior vena cava, the graft heart has pulmonary circulation and receives proper volume loading and blood flow toward the left ventricle. The graft can pump blood and is, thus, called a working heart. The heart rates and ejection fractions of the transplanted hearts were similar to those of the native hearts and also exhibited good wall motion with strains at physiological ranges.

Grafts were harvested after in-vivo CMRI assessment at end-points around Post-Operative Day (POD) 14-112, and at least five transplants were performed per end-point. The grafts were fixed in 4\% paraformaldehyde for $24 \mathrm{hrs,}$, followed by MR microscopy (MRM), histopathology, immunohistochemistry, and fluorescence microscopy examinations. Isografts served as the control groups and were exposed to the same surgical procedure and CMRI assessments as the allografts.

Animal protocols were approved by the Institutional Animal Care and Use Committee of Carnegie Mellon University. For allogenic transplantation (allograft, $n=88$ ), PVG.1U (RT1. $A^{u} B^{u} D^{u} C^{u}$ ) and PVG.R8 (RT1. $A^{a} B^{u} D^{u} C^{u}$ ) rats obtained from the University of Louisville and bred in-house were used as donors and recipients, respectively [18].

\subsection{In-Vivo Immune-Cell Labeling}

Immune cells (mainly monocytes/macrophages) were labeled in situ (12 mg Fe/kg) at POD 14 with MPIO particles containing dragon green fluorescence (Cat\# MC05F; Bangs Laboratories, Fishers, IN) according to our previous protocol [18, 19]. MPIO particles are coated with polystyrene-divinyl benzene and are not biodegradable. Thus, the internalized MPIO particles would stay in the labeled cells for the entire life cycle and the labeled cells monitored by MRI longitudinally [18].

\subsection{In-Vivo CMRI Assessment}

In order to monitor the development of CAV to capture the onset and ultimately, to identify the early stages of the disease, graft hearts were imaged by serial in-vivo CMRI to map labeled cells, starting 24 hrs before and after MPIO injection, and again around POD 28, 56, 84 and 112. The CMRI procedure was previously described [19]. Briefly, rats were intubated and ventilated with $2 \%$ isoflurane in a 2:1 $\mathrm{O}_{2}: \mathrm{N}_{2} \mathrm{O}$ gas mixture at $1.0 \mathrm{~mL} / 100 \mathrm{~g}$ body weight and 60 breaths $/ \mathrm{min}$. Rectal temperature was maintained at $36.5 \pm 0.1^{\circ} \mathrm{C}$ with a warm air system and ECG of the transplanted hearts was monitored with subcutaneous needle electrodes (SA Instruments, Stony Brook, NY) placed on the abdomen. CMRI was performed on a Bruker BioSpec 4.7-Tesla/40-cm system equipped with a $12-\mathrm{cm}, 40-\mathrm{G} / \mathrm{cm}$ shielded gradient set. Multislice ECG- and respiratory-gated $\mathrm{T}_{2}{ }_{-}$ weighted gradient-echo images were acquired with the following parameters: repetition time $=1$ respiration cycle $(1 \mathrm{~s})$; echo time $=7 \mathrm{~ms}$; field of view $(F O V)=3$ to $4 \mathrm{~cm}$; slice thickness $=1$ or 1.5 
$\mathrm{mm}$; in-plane resolution $=156 \mu \mathrm{m}$. At the designated endpoint of the study, the graft was harvested and fixed in $4 \%$ paraformaldehyde.

\subsection{In-Vivo Multiplanar Tagging MRI}

Transplanted hearts were scanned with multiplanar tagging MRI covering the whole heart volume as previously described [22]. Strains were analyzed with the HARmonic Phase method (HARP) to evaluate the regional circumferential (Ecc) and radial strain (Err) [26].

\subsection{MRM Assessment}

MRM imaging of fixed hearts was performed ex vivo using a Bruker AVANCE DRX 11.7-Tesla/89$\mathrm{mm}$ system with a Micro2.5 gradient insert as previously described [19]. High-resolution 3D gradient-echo images were acquired with the following parameters: repetition time $=500 \mathrm{~ms}$; echo time $=8 \mathrm{~ms}$; isotropic resolution $=40 \mu \mathrm{m}$.

\subsection{Pathological Analysis}

Pathology examinations were performed independently by the University of Pittsburgh Medical Center Transplantation Pathology Laboratory. Paraffin-embedded heart tissue was cut transversely at 5- $\mu \mathrm{m}$ intervals and sequential sections were stained with hematoxylin and eosin (H\&E), Perls' Prussian blue staining for iron detection, and immunohistochemical (IHC) staining for determination of monocytes/macrophages (anti-ED1, AbD Serotec, Oxford, UK, Catalog\# MCA341R), and the smooth muscle cell layer [anti-smooth muscle actin (SMA), Dako Inc., Carpinteria, CA, Catalog\# M0851] of heart vessels. ED1 is the rat homologue of human CD68. The degree of CAV was scored from 0 to 3 (Figure 1) based on the scale of mononuclear cell infiltration and the circumferential thickening affecting the lumen using a modified form of the criteria according to previous reports $[23,27]$. A grade of $0(\mathrm{G} 0)$ indicates a normal coronary artery, G1 indicates faint and limited infiltration and thickening of the vascular wall involving approximately $50 \%$ of the perimeter of the vessel, G2 indicates moderate infiltration and thickening involving between $50 \%$ and $100 \%$ of the perimeter of the vessel and, G3 indicates severe infiltration and thickening involving $100 \%$ of the perimeter of the vessel. To determine the relationship between the severity of CAV and the density of $E D 1^{+}$cells infiltrated in/around the arteries, at least 12 vessels (approximately $0.2 \mathrm{~mm}$ in diameter) per CAV grade were inspected independently by two researchers in a blinded manner using an optical microscope (Olympus PROVIS AX 70, Olympus America, Melville, NY).

\subsection{Fluorescence Microscopy Assessment}

Graft sections corresponding to hypointense regions detected by CMRI were inspected with fluorescence microscopy. Five-micrometer sections of snap-frozen allograft tissue were incubated with mouse anti-rat ED1 ${ }^{+} \mathrm{mAb}$ (AbD Serotec, Oxford, UK) for 30 minutes at room temperature in dark followed by incubation with biotinylated anti-mouse antibody and avidin D Texas red (Vector Laboratories, Peterborough, UK) for 30 minutes at room temperature in dark. Sections were then examined with dual-channel fluorescence imaging using an Olympus PROVIS AX 70 fluorescence microscope (Olympus America, Melville, NY) equipped with an automatic exposure system 
(Olympus, Tokyo), with FITC channel (495 nm excitation, $515 \mathrm{~nm}$ emission) for Dragon green (MPIO) and Cy5 channel ( $649 \mathrm{~nm}$ excitation, $668 \mathrm{~nm}$ emission) to detect Texas red in ED ${ }^{+}$cells in the same FOV.

\subsection{Quantification of MPIO-Labeled Cells and ED1+ Cells in Heart Grafts}

Phase map cross-correlation Detection and Quantification (PDQ) is a computer algorithm that automatically identifies and quantifies MPIO-labeled cells infiltrating into graft hearts [28]. PDQ was applied to 3D MRM data of heart samples with $100 \%$ volume coverage. Randomly selected graft hearts $(n=22)$ were inspected by PDQ. To confirm whether PDQ's MRI-based inspection correlated with the pathology-determined infiltration of ED1 ${ }^{+}$cells, the PDQ counting results were compared with the number of ED1 ${ }^{+}$cells counted manually on IHC sections of the heart grafts in a blinded manner. Five randomly chosen fields per section were inspected on the myocardium at the middle ventricular level. Manual counts were originally expressed as the number of cells per FOV using an eyepiece graticule and a $20 \times$ objective lens. This number was then expressed as the average number of cells $/ \mathrm{mm}^{2}$ for comparison with PDQ counts.

\subsection{Statistical Data Analysis}

Comparisons of the means of $\mathrm{ED}^{+}$cells infiltrated in grafts with different CAV grades were determined using one-way ANOVA (Excel, Microsoft, Seattle, WA). PDQ counting results of the MPIO-labeled cells were correlated with the counts of ED $1^{+}$cells in the same grafts. The trend line and the R-squared value were calculated by linear regression using Origin Pro (OriginLab Corporation, Northampton, MA). Values from multiple experiments are expressed as mean \pm SEM. Statistical significance was established at $5 \%$ probability $(p<0.05)$.

\section{Results}

\subsection{Pathological Features of the CAV Lesions Present in Our WkHt Model}

Our pre-clinical model presents the features of CAV lesions similar to clinical observations where vascular lesions are characterized by mononuclear cell infiltration, arterial wall thickening and smooth muscle cell proliferation (Figure 1). Within 3 weeks after transplantation, our pathology results found CAV lesions in some allograft hearts, which become more profound over time, up to POD 112. This relatively long time-frame to generate lesions provides us with an experimental window to observe patterns of vascular changes and identify early features of the process before vessels narrowing. The $\mathrm{H} \& \mathrm{E}$ and $\mathrm{SMA}^{+}$stained tissues slices are shown in Figure $1 \mathrm{~A}$, where isografts exposed to the same surgical procedure as allograft transplants do not exhibit histological evidence of vascular abnormalities. There are only a few mononuclear cells infiltrating and finite numbers of $\mathrm{SMA}^{+}$cells found in the isografts (Figure $1 \mathrm{~A}, 1^{\text {st }}$ column, G0). By contrast, the numbers of infiltrates with concurrent $\mathrm{SMA}^{+}$cells gradually increased in the allografts. One reason CAV is difficult to diagnose is that in its early stages, CAV does not involve all vessels. Instead it begins in some small arteries where the intimal thickening does not appear circumferentially. Within 3 weeks after transplantation, our pathology results found CAV lesions in some allograft hearts (Figure $1 \mathrm{~A}, 2^{\text {nd }}$ column, G1), which increased over time (Figure $1 \mathrm{~A}, 3^{\text {rd }}$ column, G2), until full 
involvement of the vessels at POD 112 (Figure $1 \mathrm{~A}, 4^{\text {th }}$ column, G3). These observations indicate that perivascular and focal inflammatory infiltrates appear first, followed by smooth muscle cell proliferation, which regionally affects the allograft small arteries in the early phase of CAV in our rat model, and is consistent with previous studies [18, 21].
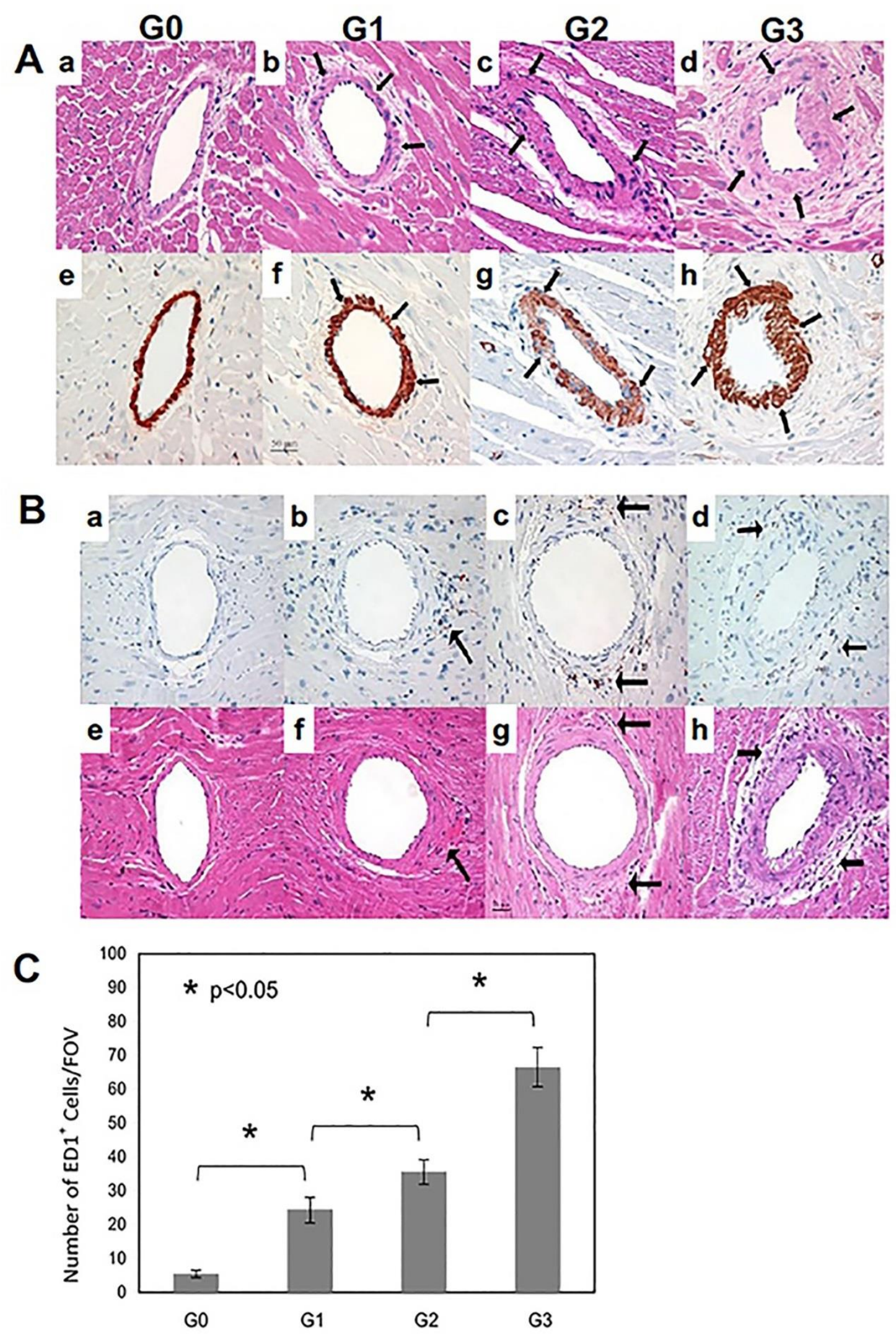
Figure 1 Pathological features of the CAV and the association between ED1+ cells and CAV lesions presented in our WkHt model. (A) Representative optical images of H\&E (a-d) and SMA (e-h, brown color) staining for grading CAV. a, e show an isograft artery on POD 95 (G0); b, f indicate a G1 allograft on POD 22 ; $c$, g indicate a G2 allograft artery on POD 60 ; and $d, h$ indicate a $G 3$ allograft artery on POD 112. Top panels are neighboring sections to bottom panels. Scale bar represents $50 \mu \mathrm{m}$. Arrows point to vascular wall thickening. (B) The pathological relationship between ED1 ${ }^{+}$cells and CAV. IHC for ED1 ${ }^{+}$ (Panels a-d) and H\&E stain for CAV pathology (Panels e-h) depicting G0, G1, G2 and G3 $\mathrm{CAV}$, respectively) show the concomitant nature of the density of $\mathrm{ED}^{+}$cells (brown color) and the severity of CAV. The arteries exhibit normal features in the isograft shown in the first column. The vascular involvement (arrows) in allograft is highly heterogeneous. It can be described as a focal vasculitis at the early phase of CAV or inflammatory cuffing of the vessel at a late stage of CAV. (C) Association between ED1 ${ }^{+}$cells and CAV. The Mean \pm SD numbers of ED1 ${ }^{+}$cells are: $5.5 \pm 1.2,24.5 \pm 3.6,35.7 \pm 3.4$ and $66.6 \pm 5.8 / F O V$ for CAV grades G0, G1, G2 and $G 3$, respectively. There is a significant association between severity of CAV and the numbers of ED $1^{+}$cells infiltrated in/around the arteries $(P<0.05)$.

IHC staining of consecutive graft sections indicated that the infiltrates consist mostly of ED $1^{+}$ cells (monocytes/macrophages, Figure 1B, a-d). The ED1 ${ }^{+}$cells in allografts, which undergo CAV, constitute more than half of the infiltrates (Figure 1B, b-d). These cells were present mainly in the adventitia of the allograft with little or no accompanying inflammation in the myocardium (Figure $1 \mathrm{~B}, \mathrm{~b}$; brown color, arrows) in the early stage. ED1 ${ }^{+}$cell accumulation was significantly less in the isograft (Figure 1, a) than in allografts with CAV (Figure 1B, b-d), though both transplants were performed under identical procedures.

To analyze the association between CAV progression and ED1 ${ }^{+}$cells, the number of ED1 ${ }^{+}$cells present around the arteries was counted per field of view (FOV) for the different stages of CAV, determined by studying consecutive slices of H\&E stained tissue (Figure 1B, e-h) and ED1 stained (Figure $1 B, a-d$ ). Our results showed that $E D 1^{+}$cell infiltration gradually increased around the vessel during the CAV progression with eventual involvement of the entire arterial tree seen in severe or very late stages of CAV. The association between ED $1^{+}$cells and vessel lesions is shown in Figure $1 C$. The mean \pm SEM numbers of ED1 ${ }^{+}$cells are: $5.5 \pm 1.2 ; 24.5 \pm 3.6 ; 35.7 \pm 3.4$; and $66.6 \pm 5.8 /$ FOV for G0, G1, G2 and G3 CAV, respectively. We found a significant association between the severity of CAV and the density of ED1 ${ }^{+}$cells $(p<0.05)$.

\subsection{CMRI Patterns of CAV Lesions and Related Confirmations}

Based on the above pathology observations, the infiltration pattern of ED1 ${ }^{+}$cells (mostly monocytes/macrophages) could be a useful indicator for CAV assessment. These cells can be visualized by CMRI when labeled with MRI contrast agents like MPIO. Areas of in-vivo $\mathrm{T}_{2}{ }^{*}$ image hypointensity (Figure $2 \mathrm{~A}$, a) result from the distribution of MPIO-labeled cells infiltrating the CAV lesions, which can be more clearly observed ex-vivo by MRM (Figure $2 A, b$ for long axis, Figure $2 A c$ for 3-D volumetric rendering, and Figure 2A, $d$ for short axis). At the early stages of CAV, CMRI reveals a few focal areas of signal hypointensity in an allograft that is undergoing CAV. Perls' Prussian blue staining of a tissue slice co-registered with the MRM image confirms these findings 
(Figure 2A, e). Areas with greater image hypointensity (Figure 2A, b-d, inserted circle) correlate well with increased iron-staining density (Figure 2A, e, inset box).

We investigated the characteristics of CAV progression via serial CMRI. Representative serial images of an allograft at different PODs are shown in Figure 2B. During the early phases of CAV, the allograft hearts display only a few spots of hypointensity from MPIO-labeled ED $1^{+}$cells (Figure $2 \mathrm{~B}, \mathrm{a})$. As CAV progresses, the same heart shows increased areas of image hypointensity, Figure 2B, panels $b-c$, indicating an increased number of MPIO-labeled cells accumulating in the rejecting tissue. It is very challenging to observe these changes in vivo by current clinical surveillance methods. The infiltrating MPIO-labeled cells were clearly seen when imaged ex vivo, where the punctuate spots of image hypointensity correspond to areas of hypointensity observed with invivo CMRI (Figure $2 \mathrm{~B}, \mathrm{C}$ ). By comparison, only very few dark spots are observed in isografts imaged both in vivo and ex vivo as illustrated in Figure $2 \mathrm{~B}$, panels e and $\mathrm{f}$, respectively.

$\mathrm{CMRI}$ is able to discriminate mild (Figure $3 \mathrm{~A}$, panels a-c) from severe (Figure 3A, panels d-f) CAV. Only a few hypointense spots were detected by in-vivo CMRI in the allograft with mild rejection (Figure 3A, a) compared with the allograft with severe CAV (Figure 3A, d).-Fluorescence microscopy performed on tissue sections which correspond to the white box on the CMRI images confirm these observations (Figure 3A, b, e). A greater number of Dragon green fluorescent spots were present in allografts with severe CAV (Figure $3 \mathrm{~A}$, panel e). Of note, these bright fluorescence spots appeared mostly around vessels. H\&E staining (Figure $3 A, c, f$ ) reveals only slight interstitial infiltration and arterial intimal thickening in the allograft with mild CAV (Figure $3 A, c$ ), but extensive changes in the allograft with severe CAV (Figure $3 A, f$ ). To confirm that the hypointense spots detected by $\mathrm{CMRI}$ were MPIO-labeled $\mathrm{ED} 1^{+}$cells, we used dual-channel fluorescence microscopy. Figure 3B, panel a, shows a representative image from a graft with severe CAV. Discrete circular spots and dark regions of hypointensity are clearly visible with MRM on most areas of the graft. Figure 3B, panels $b-d$, represent corresponding tissue sections, inspected via dual-channel fluorescence microscopy. Figure $3 B$, panel $b$ shows the Dragon green fluorescence from the MPIO particles. Figure 3B, panel $c$, is the same tissue section stained for the presence of $E D 1^{+}$cells. Figure $3 B$, panel $d$, is an overlay of panels $b$ and $c$ which clearly shows that hypointense spots in MRM image are individual or clusters of MPIO-labeled ED1+ cells though it seems more ED1+ cells than Dragon green fluorescence (MPIO particles) contenting cells.

\subsection{Correlation between MPIO-Labeled Cell Count and ED1+Cell Count}

Quantification of the correlation between MRM-detected cell infiltration and pathologistobserved $\mathrm{ED}^{+}{ }^{+}$cell infiltration was carried out in a blinded manner. The computer algorithm PDQ was applied to 3D MRM datasets of heart samples $(n=22)$ with $100 \%$ volume coverage, in order to count the number of MPIO-labeled cells or cell clusters throughout the entire heart. The MRM results correlated with histopathological inspection of the numbers of ED1 ${ }^{+}$cells infiltrated in the grafts (Figure $3 \mathrm{C}$ ). Notably, there is a strong linear correlation between the ED ${ }^{+}$cell counts and the PDQ dipole counts $\left(R^{2}=0.8075\right)$. Both of these measures correlated with increased CAV; therefore, tracking $\mathrm{ED} 1^{+}$cell counts is an excellent marker for CAV assessment by CMRI. 

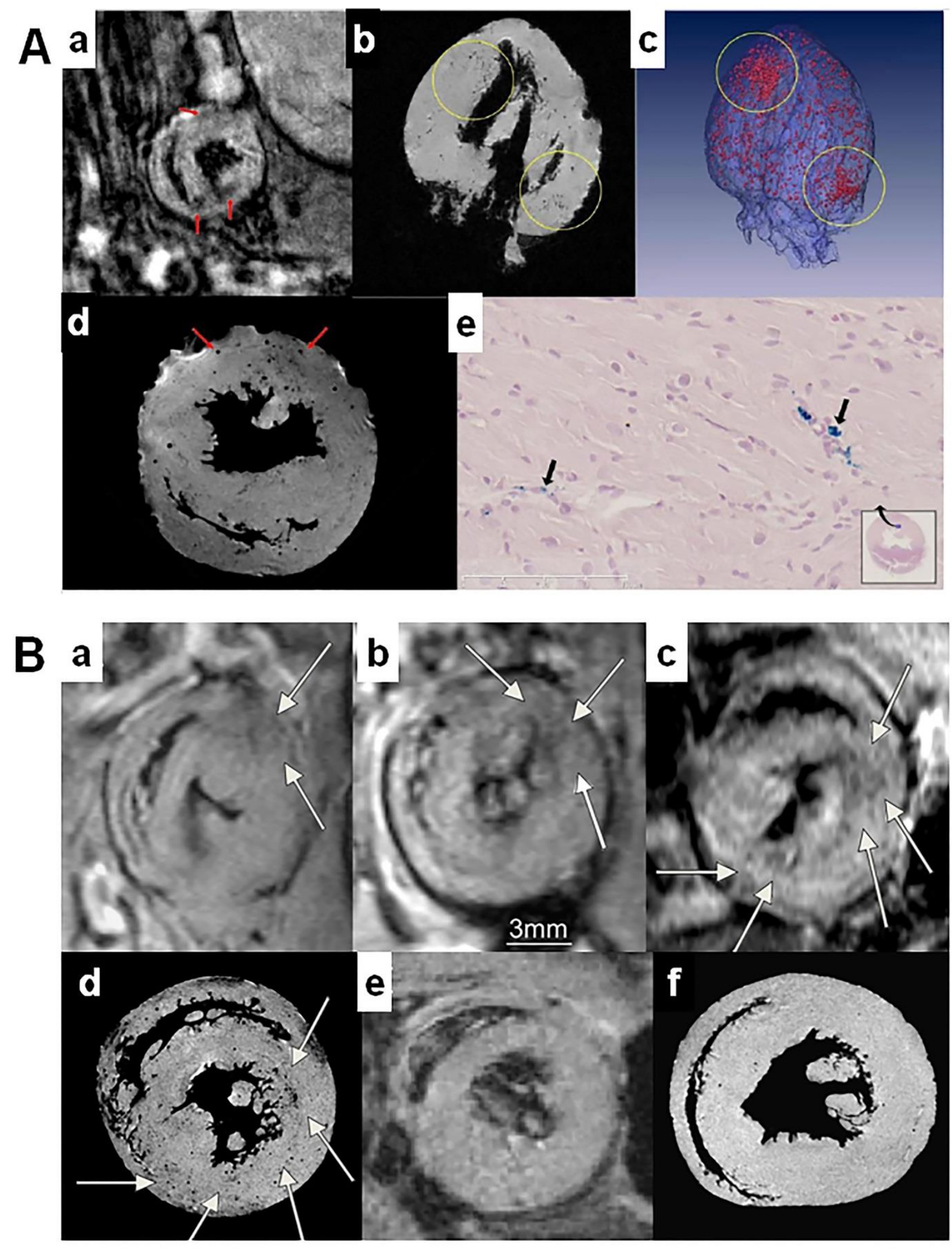

Figure 2 CMRI revealed the patterns of CAV lesions and characteristics of CAV progression. (A) In-vivo CMRI T2*-weighted image reveals heterogeneity of early CAV as hypointense signals shown in a few areas of the heart (a, arrows); (b) shows the long axis of the MRM image at 11.7 Tesla; (c) shows 3-dimensional volumetric surface rendering, inset circles show the areas with concentrated hypointensity; (d) shows the short axis of 
the MRM image; and (e) shows its co-registered with whole slice of tissue from (d) stained with Perl's Prussian blue for detecting iron (blue stain, arrows). All panels are from a single rat heart harvested on POD 94. (B) In-vivo CMRI of the same allograft at 4.7 Tesla on PODs 47 (a), 54 (b) and 81 (c). Only a few areas show hypointense spots at an early stage of CAV (a, arrows); as the disease progresses, the same image slice shown on POD 47 (a) gradually has more and larger areas containing hypointense spots (b, c). MRM at $11.7 \mathrm{~T}$ of the graft harvested on POD 81 (d) after CMRI clearly shows the punctate spots corresponding with image $c$. The hypointense spots were not observed in an isograft (e and f) on POD 99 under the same imaging conditions.
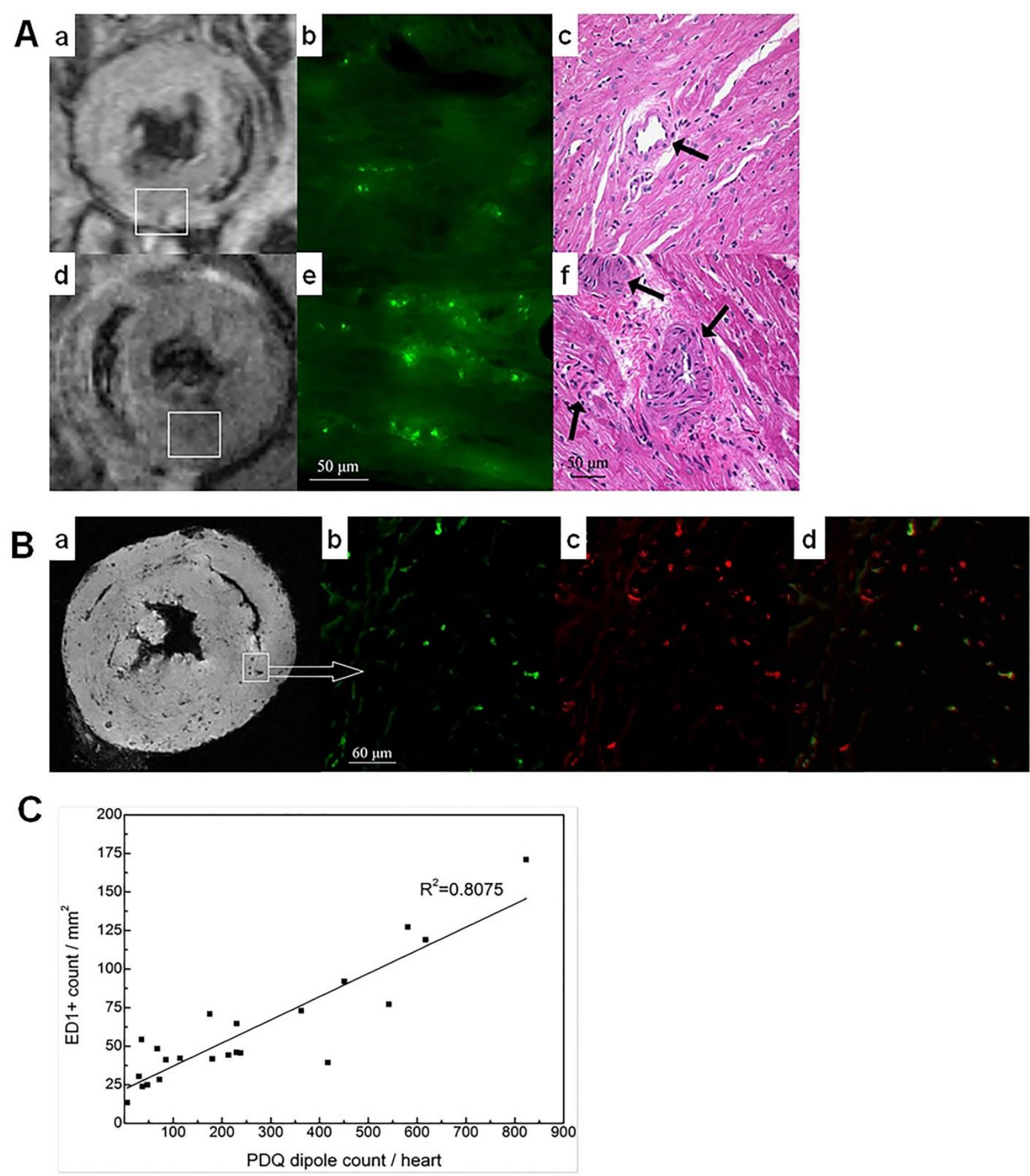
Figure 3 Correlation beteewn CMRI patterns and CAV lesions, and related confirmations. (A) few hypointense spots (within inlaid white boxes) are detected by CMRI in an allograft experiencing mild CAV (a), but a much more hypointense area appears in an allograft with severe CAV (d). Fluorescent microscope images $(b, e)$ of tissues corresponding to the boxed region in images (a) and (d) demonstrate less density of Dragon green fluorescence (MPIO particles) in the mild CAV (b) than in the severe CAV (e) and the green fluorescence appears mostly around the vessels; (c) and (f) are H\&E stains of neighboring sections of (b) and (e), respectively. Scale bar represents $50 \mu \mathrm{m}$. (B) Hypointense spots in CMRI are individual or clusters of MPIO-labeled ED1 ${ }^{+}$cells. Discrete circular spots and serial dark regions of hypointensity are clearly observed by MRM at 11.7 $\mathrm{T}$ (a) on a representative allograft with severe CAV. Dual-channel fluorescence microscopy indicates MPIO particles (b, Dragon-green, excitation: $488 \mathrm{~nm}$ ) and ED1 ${ }^{+}$cells (c, Texas red, excitation: $588 \mathrm{~nm}$ ) on the same tissue section from the boxed region in image (a). Panel (d) is an overlay image of panels (b) and (c). Scale bar represents $60 \mu \mathrm{m}$. (c) The PDQ algorithm's whole-heart counting results for MPIO-labeled cells (and cell clusters) are compared with the counts of $E D 1^{+}$cells within histological sections from randomly selected graft hearts $(n=22)$. The trend line and $R$-squared value were calculated by linear regression using Origin Pro (OriginLab Corporation, Northampton, $\mathrm{MA})$.

\subsection{Assessment of Ventricular Function of CAV}

To access the ventricular function of CAV, we performed multiplanar tagging MRI covering the whole heart volume [22] followed by strain analysis for quantifying circumferential and radial strain. Figure 4 shows an allograft heart subjected to simultaneous in vivo cellular and tagging MRI on PODs 47, 54, 73, and 81. Two out of 7 short-axis slices from the heart volume are shown. Top panels (Figure $4 \mathrm{~A}, \mathrm{C}, \mathrm{E}, \mathrm{G}, \mathrm{I}, \mathrm{K}, \mathrm{M}, \mathrm{O}$ ) show $\mathrm{T}_{2}{ }^{*}$-weighted cellular $\mathrm{MRI}$ after one single bolus injection of MPIO 2 weeks after transplantation. On POD 47 (Figure 4, A and I), very few MPIOlabeled infiltrated macrophage foci are detected (white arrow-heads). As the CAV progressed over time, more MPIO-labeled macrophages were recruited to the rejection site without further injection. On POD 81 (Figure $4 \mathrm{G}$ and O), multiple MPIO-positive cellular foci can be clearly seen (white arrow-heads). While the reduction in ejection fraction (EF, 61.1\% on POD 47 and $51.5 \%$ on POD 81) was mild, heterogeneous decrease in circumferential strain (Figure 4 lower panels, B, D, F, $\mathrm{H}, \mathrm{J}, \mathrm{L}, \mathrm{N}, \mathrm{P}$ ) is seen in parts of the left ventricle. Circumferential strain was largely normal on POD 47 (Figure $4 \mathrm{~B}$ and J), whereas the regions with compromised strain became larger on POD 81 (Figure $4 \mathrm{H}$ and $\mathrm{P}$ ). The ventricular regions that displayed compromised circumferential strain (Figure 4 white arrows in lower panels $B, D, F, H, J, L, N, P$ ) correlated with the regions with MPIOlabeled macrophage infiltrated foci (Figure 4 white arrows in top panels, A, C, E, G, I, K, M, O). Our data show that in our allograft heterotopic CAV model, the immune cell infiltration is spatially heterogenous. The ventricular regions with more cellular infiltration exhibited compromised regional strain. The simultaneous cellular and strain MRI can be more sensitive in detecting the regional immune-cell infiltration and the regional wall abnormality than the systolic dysfunction by EF. 


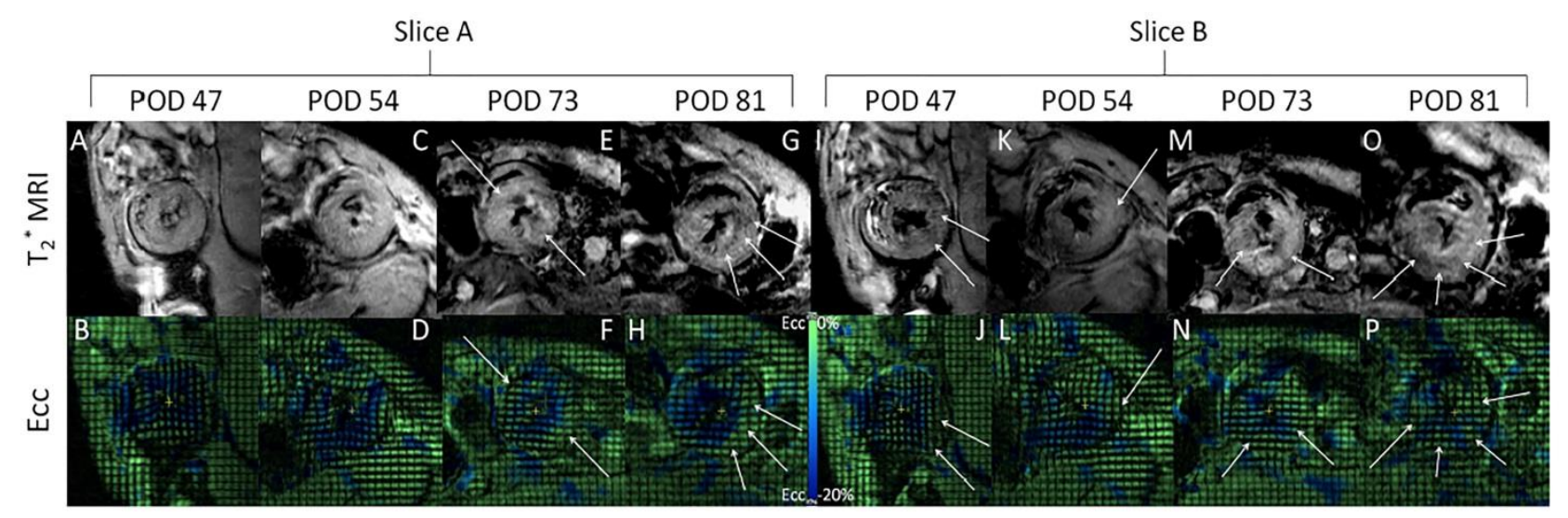

Figure 4 Simultaneous cellular and strain MRI for a heterotopic allograft working heart model, showing two imaging slices out of the whole heart volume. (Top panels- A, C, E, G, $\mathbf{I}, \mathbf{K}, \mathbf{M}, \mathbf{O}) \mathrm{T}_{2}{ }^{*}$-weighted cellular MRI after a single bolus MPIO injection. (Lower panels B, D, F, H, J, L, N, P) Circumferential strain (Ecc) map at the end-systole derived from tagging MRI acquired at the same imaging session on the same PODs as the cellular MRI. The white arrowheads point to comparable regions on the cellular and strain MRI with cellular infiltration foci and compromised Ecc.

\section{Discussion}

The present study illustrates the feasibility of using MPIO-labeled ED1 ${ }^{+}$cells as a marker for the exploration and serial characterization of CAV in vivo by CMRI. We have monitored the status of the entire heart graft to identify the early indicators of CAV before irreversible damage occurred. We believe that this is the first pre-clinical in vivo study describing the features and patterns of CAV lesion development, with information on the entire heart, which may be important to investigate the early signs of CAV.

The pathological appearance of CAV is characterized mainly by circumferential intimal thickening of the entire arterial tree of the graft $[7,8]$. But, its early signs or indicators have not yet been fully investigated in vivo. Previous studies have reported the preservation of luminal diameter at the early stages of CAV in both patients [3] and animal models [9, 10], our study also did not observe luminal narrowing in the early stages of CAV (Figure 1A). These observations may partially explain why coronary angiography can overlook the early changes in arteries, whereas IVUS is more sensitive in detecting graft coronary artery disease [6]. So far, IVUS cannot visualize the entire arterial system and allows only for the examination of proximal large arteries [5]. Recent insights into arterial remodeling have implicated the adventitial layer as being an important modulator of remodeling through its interactions with the media and intima [29]. In contrast, cellular MRI provides a method for non-invasive serial monitoring of the patterns of MPIO-labeled ED1 ${ }^{+}$cell infiltration, which are considered the final effectors for CAV [8, 9], over the entire heart. Therefore, CMRI may be an excellent tool for screening asymptomatic patients in order to provide important information in addition to angiography and IVUS on the management of patients after transplantation.

Clinical studies with MRI indicated that CMRI for detecting CAV can be more accurate by using noninvasive CMR-based absolute myocardial blood flow assessment than with invasive coronary 
angiography [30]. Investigations also reported the ability of Strain-Encoded magnetic resonance imaging (MRI) to detect CAV in heart transplantation recipients [31, 32]. It highlights the role of non-invasive CMRI for the early detection of CAV rather than coronary angiography and IVUS, because CMRI allows for the noninvasive and reproducible assessment of cardiac anatomy, deformation and function, perfusion, viability and if required metabolism and tissue characterization. Our previous study on the detection of acute cardiac rejection showed that MRI offers great potential to look beyond the temporal-spatial distribution of cells, to also provide multimodal information on cell function and composition [22]. Further development of integrated multi-parameter cellular and functional MRI modalities could provide a more comprehensive early diagnosis of CAV. Patients would benefit from a longitudinal evaluation by multiple modalities for early identification of lesions before vessels changes become irreversible.

There are many advantages to using superparamagnetic iron-oxide particles for cell-tracking studies, and the development of biodegradable iron-oxide particles suitable for clinical use is underway [33,34]. Our results are consistent with previous studies, in which these particles do not appear to have any long-term toxic effects on the organism and can remain in labeled cells after multiple cell divisions [18, 35]. One of the challenges for cellular MRI using MPIO is the quantification of the signal intensity. Recently, many methodologies have been developed for quantification of iron-labeled cells [28, 36, 37]. In this study, computational PDQ quantification of MPIO-labeled cells and cell clusters using 3D MRM data was found to be well correlated with the direct counting of $\mathrm{ED}^{+}{ }^{+}$cells in graft tissue. It suggests that the PDQ algorithm (approximately 15 min of investigator time per heart sample) may be useful for approximating the degree of ED $1^{+}$cell infiltration present, while also providing computational reproducibility and significant time savings relative to manual histological inspection. This approach promises to provide a new method for quantification of iron-labeled cells.

Mounting evidence now indicates that innate immune cells play important roles in the acute and chronic rejection of organ allografts $[18,19,38]$. Emerging data indicates that methods targeting immune-cell production, such as selective cytokines, cellular receptors, and antibodies, would ameliorate the initial steps in the development of CAV $[4,8]$, and the advance of immunosuppressive drugs has led to the success of suppression of T cells [38], but the redundancy intrinsic to the immune system does not easily lend itself to a single target approach [39]. Characterization of these cells in an allograft undergoing CAV may provide insights into lesion onset and related pathophysiological events. In current clinical situation, acute cellular rejection mostly occurs during the first six months following transplantation. Thus, systemically monitoring MPIO-labeled innate immune cells, mainly macrophages, could be a good target for in-vivo imaging to explore the early features during the development of CAV and its eventual progression, prior to arterial wall thickening and luminal narrowing by non-invasive CMRI with whole-heart information. We have observed that the macrophage infiltration patterns are different between acute cellular rejection and CAV. Acute cellular rejection is characterized by progressive patches of hypointensity caused by the infiltration of MPIO-labeled macrophages [22]. However, CAV is characterized by a gradual accumulation of foci hypointensity caused by the accumulation of MPIO-labeled macrophages (Figure 2B). As CAV progresses, the same MRI image slice gradually has more and larger areas containing hypointense spots (Figures $2 \mathrm{~B}$ a-c). Thus, we believe this work has provided experimental evidence to show that the macrophage-infiltration pattern is a useful index for monitoring graft rejection status. Future studies utilizing this working heart model 
could be used to investigate the pathophysiological mechanisms related to CAV and therapeutic interventions, in addition to further development of MRI-based modalities for the diagnosis of cardiac diseases.

\section{Conclusions}

This study demonstrates the feasibility of using MPIO-labeled ED1+ cells as a marker for exploration and serial characterization of CAV by MRI. We monitored the status of the entire heart graft to identify the early indicators of CAV in a pre-clinical model of rat heart transplantation in more than one hundred heart grafts. Our study has evealed that: (1) the infiltrates are mostly ED1+ cells and their density is significantly correlated with the severity of CAV ( $<<0.05)$; (2) CMRI shows that the areas of $\mathrm{T}_{2}{ }^{*}$-weighed image hypointensity revealing the infiltrating iron-labeled macrophages increase in number as CAV progresses; and (3) MPIO-labeled cells counted from magnetic resonance microscopy (MRM) images throughout each entire heart by a computer algorithm as well as those counted by immunohistochemical inspection of ED1+ cell infiltration exhibit a strong correlation $\left(R^{2}=0.8075\right)$. Our results highlight that CMRI not only can detect CAV early, but also can assess CAV before vessel narrowing or arterial wall thickening has developed, a period where current clinical detection methods can diagnose CAV, but where it may be too late for successful treatment. We believe that this is the first pre-clinical in-vivo study describing the features and patterns of CAV lesion development.

\section{Acknowledgments}

We greatly appreciate Mr. Brent Barbe for excellent assistance with the animals.

\section{Author Contributions}

QY, YW and $\mathrm{CH}$ designed the study, analyzed the data, and wrote the manuscript with input from all the coauthors. $Q Y$ and $Y W$ equally contributed to this manuscript. QY carried out the heart transplantation surgery and pathology studies. $\mathrm{YW}, \mathrm{LF}, \mathrm{FY}, \mathrm{HZ}, \mathrm{TH}$, and DE carried out the MRI studies. PM carried out the computer algorithm PDQ study. $\mathrm{CH}$ supervised the entire project.

\section{Funding}

This work was supported by research grants from the National Institutes of Health (R01HL081349 and P41EB-001977 to C.H.). L.L. was supported in part by a Postdoctoral Fellowship awarded by the American Heart Association (10POST3010016).

\section{Competing Interests}

The authors have declared that no competing interests exist.

\section{References}

1. Alraies MC, Eckman P. Adult heart transplant: Indications and outcomes. J Thorac Dis. 2014; 6: $1120-1128$. 
2. Yusen RD, Christie JD, Edwards L, Kucheryavaya AY, Benden C, Dipchand Al, et al. The Registry of the International Society for Heart and Lung Transplantation: Thirtieth adult lung and heart-lung transplant report--2013; focus theme: Age. J Heart Lung Transplant. 2013; 32: 965978.

3. Stehlik J, Edwards LB, Kucheryavaya AY, Aurora P, Christie JD, Kirk R, et al. The Registry of the International Society for Heart and Lung Transplantation: Twenty-seventh official adult heart transplant report-2010. J Heart Lung Transplant. 2010; 29: 1089-1103.

4. Dhaliwal A, Thohan V. Cardiac allograft vasculopathy: The Achilles' heel of long-term survival after cardiac transplantation. Curr Atheroscler Rep. 2006; 8: 119-130.

5. Logani S, Saltzman HE, Kurnik P, Eisen HJ, Ledley GS. Clinical utility of intravascular ultrasound in the assessment of coronary allograft vasculopathy: A review. J Interv Cardiol. 2011; 24: 914.

6. Kass M, Haddad H. Cardiac allograft vasculopathy: Pathology, prevention and treatment. Curr Opin Cardiol. 2006; 21: 132-137.

7. Hutchinson IV. Cardiac allograft vasculopathy-the cellular attack. Z Kardiol. 2000; 89: IX16IX20.

8. Mitchell RN. Graft vascular disease: Immune response meets the vessel wall. Annu Rev Pathol. 2009; 4: 19-47.

9. Russell PS, Chase CM, Winn HJ, Colvin RB. Coronary atherosclerosis in transplanted mouse hearts. I. Time course and immunogenetic and immunopathological considerations. Am J Pathol. 1994; 144: 260-274.

10. Russell ME, Adams DH, Wyner LR, Yamashita Y, Halnon NJ, Karnovsky MJ. Early and persistent induction of monocyte chemoattractant protein 1 in rat cardiac allografts. Proc Natl Acad Sci U S A. 1993; 90: 6086-6090.

11. Munder $M$, Mallo $M$, Eichmann K, Modolell $M$. Murine macrophages secrete interferon gamma upon combined stimulation with interleukin (IL)-12 and IL-18: A novel pathway of autocrine macrophage activation. J Exp Med. 1998; 187: 2103-2108.

12. Underhill DM, Bassetti M, Rudensky A, Aderem A. Dynamic interactions of macrophages with T cells during antigen presentation. J Exp Med. 1999; 190: 1909-1914.

13. Ho C, Hitchens TK. A non-invasive approach to detecting organ rejection by MRI: Monitoring the accumulation of immune cells at the transplanted organ. Curr Pharm Biotechnol. 2004; 5: 551-566.

14. Ye $Q$, Yang $D$, Williams $M$, Williams $D S$, Pluempitiwiriyawej $C$, Moura JM, et al. In vivo detection of acute rat renal allograft rejection by MRI with USPIO particles. Kidney Int. 2002; 61: 1124-1135.

15. Himmelreich $U$, Dresselaers T. Cell labeling and tracking for experimental models using magnetic resonance imaging. Methods. 2009; 48: 112-124.

16. Bulte JWM. In vivo MRI cell tracking: Clinical studies. AJR Am J Roentgenol. 2009; 193: 314325.

17. Liu L, Ye Q, Wu Y, Hsieh WY, Chen $\mathrm{CL}$, Shen $\mathrm{HH}$, et al. Tracking T-cells in vivo with a new nanosized MRI contrast agent. Nanomedicine. 2012; 8: 1345-1354.

18. Ye Q, Wu YL, Foley LM, Hitchens TK, Eytan DF, Shirwan H, et al. Longitudinal tracking of recipient macrophages in a rat chronic cardiac allograft rejection model with noninvasive 
magnetic resonance imaging using micrometer-sized paramagnetic iron oxide particles. Circulation. 2008; 118: 149-156.

19. Wu YL, Ye Q, Foley LM, Hitchens TK, Sato K, Williams JB, et al. In situ labeling of immune cells with iron oxide particles: An approach to detect organ rejection by cellular MRI. Proc Natl Acad Sci U S A. 2006; 103: 1852-1857.

20. Hitchens TK, Ye Q, Eytan DF, Janjic JM, Ahrens ET, Ho C. 19F MRI detection of acute allograft rejection with in vivo perfluorocarbon labeling of immune cells. Magn Reson Med. 2011; 65: 1144-1153.

21. Shirwan H, Mhoyan A, Yolcu ES, Que X, Ibrahim S. Chronic cardiac allograft rejection in a rat model disparate for one single class I MHC molecule is associated with indirect recognition by CD4(+) T cells. Transpl Immunol. 2003; 11: 179-185.

22. Wu YL, Ye Q, Sato K, Foley LM, Hitchens TK, Ho C. Noninvasive evaluation of cardiac allograft rejection by cellular and functional cardiac magnetic resonance. JACC Cardiovasc Imaging. 2009; 2: 731-741.

23. Adams DH, Tilney NL, Collins JJ, Jr., Karnovsky MJ. Experimental graft arteriosclerosis. I. The Lewis-to-F-344 allograft model. Transplantation. 1992; 53: 1115-1119.

24. Hasegawa T, Visovatti SH, Hyman MC, Hayasaki T, Pinsky DJ. Heterotopic vascularized murine cardiac transplantation to study graft arteriopathy. Nat Protoc. 2007; 2: 471-480.

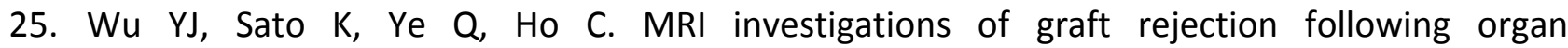
transplantation using rodent models. Methods Enzymol. 2004; 386: 73-105.

26. Osman NF, Prince JL. Visualizing myocardial function using HARP MRI. Phys Med Bio. 2000; 45 : 1665-1682.

27. Mehra MR, Crespo-Leiro MG, Dipchand A, Ensminger SM, Hiemann NE, Kobashigawa JA, et al. International Society for Heart and Lung Transplantation working formulation of a standardized nomenclature for cardiac allograft vasculopathy-2010. J Heart Lung Transplant. 2010; 29: 717-727.

28. Mills PH, Hitchens TK, Foley LM, Link T, Ye Q, Weiss CR, et al. Automated detection and characterization of SPIO-labeled cells and capsules using magnetic field perturbations. Magn Reson Med. 2012; 67: 278-289.

29. Mitchell RN, Libby P. Vascular remodeling in transplant vasculopathy. Circ Res. 2007; 100: 967-978.

30. Miller CA, Sarma J, Naish JH, Yonan N, Williams SG, Shaw SM, et al. Multiparametric cardiovascular magnetic resonance assessment of cardiac allograft vasculopathy. J Am Coll Cardiol. 2014; 63: 799-808.

31. Korosoglou G, Osman NF, Dengler TJ, Riedle N, Steen H, Lehrke S, et al. Strain-encoded cardiac magnetic resonance for the evaluation of chronic allograft vasculopathy in transplant recipients. Am J Transplant. 2009; 9: 2587-2596.

32. Erbel C, Mukhammadaminova N, Gleissner CA, Osman NF, Hofmann NP, Steuer C, et al. Myocardial perfusion reserve and strain-encoded $C M R$ for evaluation of cardiac allograft microvasculopathy. JACC Cardiovasc Imaging. 2016; 9: 255-266.

33. McAteer MA, Akhtar AM, von zur Muhlen C, Choudhury RP. An approach to molecular imaging of atherosclerosis, thrombosis, and vascular inflammation using microparticles of iron oxide. Atherosclerosis. 2010; 209: 18-27. 
34. Chen $\mathrm{CL}$, Zhang $\mathrm{H}, \mathrm{Ye} \mathrm{Q}$, Hsieh $\mathrm{WY}$, Hitchens $\mathrm{TK}$, Shen $\mathrm{HH}$, et al. A new nano-sized iron oxide particle with high sensitivity for cellular magnetic resonance imaging. Mol Imaging Biol. 2011; 13: 825-839.

35. Shapiro EM, Skrtic S, Koretsky AP. Sizing it up: Cellular MRI using micron-sized iron oxide particles. Magn Reson Med. 2005; 53: 329-338.

36. Kuhlpeter R, Dahnke H, Matuszewski L, Persigehl T, von Wallbrunn A, Allkemper T, et al. R2 and R2* mapping for sensing cell-bound superparamagnetic nanoparticles: In vitro and murine in vivo testing. Radiology. 2007; 245: 449-457.

37. Varma G, Clough RE, Acher P, Senegas J, Dahnke H, Keevil SF, et al. Positive visualization of implanted devices with susceptibility gradient mapping using the original resolution. Magn Reson Med. 2011; 65: 1483-1490.

38. Wood KJ, Goto R. Mechanisms of rejection: Current perspectives. Transplantation. 2012; 93 : 1-10.

39. Cravedi P, Heeger PS. Immunologic monitoring in transplantation revisited. Curr Opin Organ Transplant. 2012; 17: 26-32.

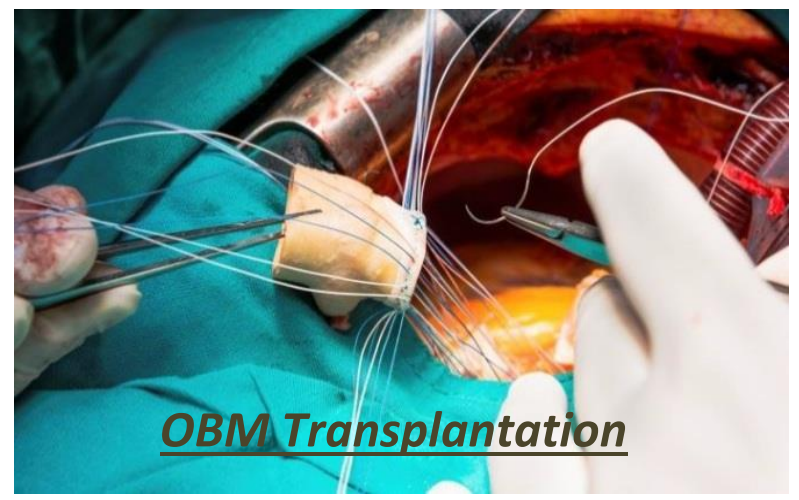

Enjoy OBM Transplantation by:

1. Submitting a manuscript

2. Joining in volunteer reviewer bank

3. Joining Editorial Board

4. Guest editing a special issue

For more details, please visit: http://www.lidsen.com/journals/transplantation 